\title{
UCHL1/PGP 9.5 Dynamic in Neuro-Immune-Cutaneous Milieu: Focusing on Axonal Nerve Terminals and Epidermal Keratinocytes in Psoriatic Itch
}

\author{
Piotr Kupczyk $\mathbb{D},{ }^{1,2}$ Adam Reich $\mathbb{D}^{3},{ }^{3}$ Mariusz Gajda $\mathbb{D}^{4},{ }^{4}$ \\ Marcin Hołysz, ${ }^{5}$ Edyta Wysokińska, ${ }^{6}$ Maria Paprocka, ${ }^{7}$ \\ Dmitry Nevozhay, ${ }^{8,9}$ Grzegorz Chodaczek, ${ }^{10}$ Paweł P. Jagodziński $\left(\mathbb{D},{ }^{5}\right.$ \\ Piotr Ziółkowski, ${ }^{1}$ and Jacek C. Szepietowski ${ }^{11}$
}

${ }^{1}$ Department of Pathomorphology, Faculty of Medicine, Silesian Piast Wroclaw Medical University, Wroclaw, Poland

${ }^{2}$ Laboratory of Immunogenetics and Tissues Immunology, Ludwik Hirszfeld Institute of Immunology and Experimental Therapy, Polish Academy of Sciences, Wroclaw, Poland

${ }^{3}$ Department of Dermatology, Institute of Experimental and Clinical Medicine, Faculty of Medicine, University of Rzeszow, Rzeszow, Poland

${ }^{4}$ Department of Histology, Medical Collage, Jagiellonian University, Krakow, Poland

${ }^{5}$ Department of Biochemistry and Molecular Biology, Karol Marcinkowski Medical University of Poznan, Poznan, Poland

${ }^{6}$ Laboratory of Tumor Molecular Immunobiology, Ludwik Hirszfeld Institute of Immunology and Experimental Therapy, Polish Academy of Sciences, Wroclaw, Poland

${ }^{7}$ Laboratory of Glycobiology and Cell Recognitions, Ludwik Hirszfeld Institute of Immunology and Experimental Therapy, Polish Academy of Sciences, Wroclaw, Poland

${ }^{8}$ School of Biomedicine, Far Eastern Federal University, Vladivostok, Russia

${ }^{9}$ Department of Imaging Physics, MD Anderson Cancer Center, The University of Texas, Houston, Texas, USA

${ }^{10}$ Confocal Microscopy Laboratory, Wroclaw Research Centre EIT+, Wroclaw, Poland

${ }^{11}$ Department of Dermatology, Venereology and Allergology, Silesian Piast Wroclaw Medical University, Wroclaw, Poland

Correspondence should be addressed to Piotr Kupczyk; piotr.kupczyk.81@gmail.com

Received 29 January 2018; Revised 28 May 2018; Accepted 7 June 2018; Published 25 July 2018

Academic Editor: Maxim E. Darvin

Copyright (C) 2018 Piotr Kupczyk et al. This is an open access article distributed under the Creative Commons Attribution License, which permits unrestricted use, distribution, and reproduction in any medium, provided the original work is properly cited.

Psoriasis is an immunogenetic skin disease manifesting as plaque lesions on the skin. Patients with psoriasis frequently suffer from itch, an unpleasant sensation causing a desire to scratch. Psoriatic itch is mainly transmitted by unmyelinated C-fibers; however, the exact molecular mechanism of psoriatic itch is still unexplained. Protein gene product 9.5 (PGP 9.5) is a panneurological marker commonly used for analysis of peripheral peptidergic and nonpeptidergic nerves and identification of cutaneous neuroimmune-endocrine cells. However, some studies suggested that nonneuronal cells, like keratinocytes, may also express PGP 9.5. This phenomenon might be linked with impaired axonal transport, keratinocyte injury, or dysfunctions of neuro-immune-cutaneous connections. The aim of this study was to analyze the expression of PGP 9.5 in psoriatic skin. We observed significantly altered density of PGP 9.5-positive axonal nerve terminals in pruritic lesional $(\mathrm{p}=0.04)$ and nonlesional psoriatic skin ( $\mathrm{p}>0.001)$ compared with controls. In contrast, no significant differences were observed between psoriatic skin without itch and controls. Furthermore, PGP 9.5 expression by suprabasal keratinocytes (SBKs) was significantly increased in itchy skin lesions ( $p=0.007$ ) compared to skin without itch, and a positive correlation was observed between PGP 9.5 expression and itch intensity $(r=0.64 ; p=0.02)$. Our findings indicate changes in peripheral innervations and psoriatic keratinocytes, which may influence neuro-immune-cutaneous homeostasis and modulate itch transmission. 


\section{Introduction}

Psoriasis is an immunogenetic skin disease, in which neuroinflammation is believed to be an important pathogenic element [1]. Neuroinflammation is considered as one of the possible mechanisms which might enhance itch perception, an unpleasant sensation causing a desire to scratch $[2,3]$. The neurobiological background of itch in psoriasis is still poorly understood; however abnormalities in peripheral nervous system (PNS) are considered as one of the possible mechanisms [4]. Itch is mainly mediated by unmyelinated C-fibers, which originate from dorsal root ganglion (DRG) neurons [5]. All (peptidergic and nonpeptidergic) PNS structures could be visualized by a neurobiological marker: protein gene product 9.5 (PGP 9.5), one of the most abundant proteins of central nervous system (CNS). Its quantity constitutes up to $3-5 \%$ of the total brain protein amount $[6,7]$. PGP 9.5 is a ubiquitin carboxyl-terminal hydrolase L1 (UCHL1) with dual hydrolase and ligase activity and molecular weight of $27 \mathrm{kDa}$, encoded by UCHL-1 gene $[6,8]$. PGP 9.5 is also a deubiquitinating enzyme (DUB) participating in posttranslational protein modification by adding to and removing ubiquitin from polyubiquitin chains [6]. Abnormalities in recycling or prevention of ubiquitinated proteins from the proteosomal degradation pathways are engaged in several neuro-immune-related diseases $[6,8]$. For example, the loss of PGP 9.5 protein is linked with decreased mono-Ub pool and results in abnormal function of postsynaptic compartments in PNS terminals $[9,10]$. In turn, mutations in UCHL1 gene are associated with Parkinson disease, engaged in motor neuron circuitry and pain-related dysfunctions $[6,11]$. In contrast, overexpression of UCHL1/PGP9.5 was documented as protective factor, which delays progression of Alzheimer disease [12]. Moreover, UCHL1/PGP 9.5 abnormalities were also linked with cancers, innate and adaptive mechanisms of immune response, axonal transport, traumatic brain injury, and recessive general-body neurodegeneration $[6,8]$.

In the skin, the UCHL1/PGP 9.5 is strictly connected with nerve terminals; however, several studies also demonstrated its expression by neuro-immune-endocrine cells of the skin [13]. Basal and suprabasal keratinocytes, melanocytes, and Merkel and Langerhans cells may contain UCHL1/PGP 9.5 [13-15]. UCHL1/PGP 9.5 comes from two independent expression sources, primarily from CNS, but secondarily from cutaneous-derived sources, and both meet in the skin [14-17]. Thus, UCHL1/PGP 9.5 seems to well fitful criteria of a marker, which could identify the nonneuronal "synapticlike" connections proposed by Boulais and Misery [18]. Several studies demonstrated modification of PGP 9.5 axonal nerve terminals and neuro-immune-endocrine cells in the skin during chronic cutaneous neuroinflammation [19]. Those abnormalities provide induction of molecular changes, which in advanced skin lesions might resemble immunemediated tissue injury and engage psoriatic keratinocytes in transmission of itch [20-24].

We aimed to investigate the connection between skin innervations in psoriatic individuals and itch sensation. We also have tried to elaborate the potential role of UCHL1 gene as a new neurobiological marker of itch.

\section{Material}

2.1. Patients. Twenty psoriatic patients (20\% females and $80 \%$ males) were included into the study. Their age ranged from 24 to 65 years (mean $44.1 \pm 12.5$ years). None of the included patients had received any local or systemic therapies for at least three months prior to entering the study. Individuals with other cutaneous or systemic diseases which might interfere with study results were excluded. Patients were divided into two groups in relation to their itch status: with (Itch+) and without (Itch-) itch ( $n=14$ and $n=6$, respectively). The control group consisted of 20 healthy individuals $(60 \%$ females and $40 \%$ males) who underwent a routinely scheduled correction during plastic surgery. The age of control group ranged from 30 to 79 years (mean $56.3 \pm 13.0$ years). All patients provided written informed consent, while study protocol was approved by the Ethics Committee at the Wroclaw Medical University (decision 669/2011) and was carried out in accordance with the principles of the Helsinki Declaration.

2.2. Reference Material. The total brain RNA (Agilent Technologies, USA) from human postmortem and human brain hippocampus (Clontech Laboratories, USA) were used for UCHL1 gene transcript and PGP 9.5 protein detection.

\section{Methods}

3.1. Clinical Parameters. Psoriasis Area Severity Index (PASI) was used as the parameter to assess the severity of psoriasis. The mean PASI in analyzed patients was $15.7 \pm 10.2$ points (range: 6.4-44.0 points). The Visual Analogue Scale (VAS) score was used for evaluation of maximal itch intensity within 24 hours prior to skin biopsy (mean: $3.9 \pm 2.8$ points; range: 0-8 points).

3.2. Skin Biopsies: Preservation and Fixation. The $5 \mathrm{~mm}$ punch biopsies were taken from nonlesional and lesional skin of patients with psoriasis. Using sterile scissors biopsies were divided and primary one was immediately preserved in RNAlater (Qiagen, USA) solution and transferred to the $-80^{\circ} \mathrm{C}$. The second part of a biopsy was fixed in $4 \%$ paraformaldehyde (PFA) solution in $0.1 \mathrm{M}$ phosphatase buffer saline (PBS) for 12 hours and transferred to the $30 \%$ sucrose $(\mathrm{POCH}$, Poland) solution in $133 \mathrm{mM}$ of Sörensen buffer for another 24 hours at $4^{\circ} \mathrm{C}$. After fixation, biopsies were immediately immersed in optimum cutting temperature medium (O.C.T., CellPath, United Kingdom) and $15 \mu \mathrm{m}$ frozen skin sections were prepared (Superfrost Ultra Plus, Thermo Scientific ${ }^{\mathrm{TM}}$, Germany) using cryostat (Leica, Germany) and stored at $20^{\circ} \mathrm{C}$ for further analysis.

3.3. Cell Cultures. Human immortalized keratinocyte cell line HaCaT (ACTT, USA) were cultured in DMEM (Biowest, USA) growth medium supplemented with 10\% FBS (Gibco, USA) and 1\% PSA (Sigma-Aldrich, USA) solution at $37^{\circ} \mathrm{C}$ in a humidified atmosphere of $5 \% \mathrm{CO}_{2}$ and $95 \%$ air. Cells after $70-80 \%$ confluence were incubated with $0.05 \%$ trypsin/EDTA (IITD PAN, Wroclaw, Poland) for 5 min., passaged, seeded at $2 \times 10^{5}$ density in 6 -well plate, and preserved 
for further experiments. For gene expression analyses, cells were suspended in $1 \mathrm{ml}$ of Trizol (Invitrogen, USA) and immediately transferred to $-80^{\circ} \mathrm{C}$. For protein analysis, cells were lysed using $1 \mathrm{ml}$ of RIPA buffer and transferred to $-20^{\circ} \mathrm{C}$. Finally, for immunofluorescence imaging, cells were seeded at 96-well plate and fixed in 4\% PFA for $10 \mathrm{~min}$. at RT and $100 \mu \mathrm{l}$ of PBS per well was added before immunofluorescence protocol.

3.4. RNA isolation and Reverse Transcription. The total RNA was isolated from skin biopsies embedded in RNAlater (Ambion, USA). The skin fragments were homogenized in Trizol (Invitrogen, USA) and introduced to the Tissue Fibrous Kit (Qiagen, USA) protocol. The DN-ase I treatment was applied to exclude possible contaminations and prevent hybridization of primers and probes to gDNA. Additionally, commercially available One-Step PCR Removal Inhibitor Kit (Zymo Research, USA) was also used for purification of RNA from possible PCR skin inhibitors. The quality and quantity of RNA after extraction were estimated using Nanodrop (Eppendorf, Germany) and only samples with 260/280 nm absorbance coefficient in the range between 1.8 and 2.1 were further used. The RNA samples were incubated for 10 minutes at $65^{\circ} \mathrm{C}$ and immediately transferred on ice to minimize formation of RNA secondary and tertiary structures before reverse transcription. The $500 \mathrm{ng}$ of total RNA was reverse transcribed on cDNA using First-Strand cDNA Synthesis Kit for RT-PCR (Roche, Germany).

3.5. Probe and Primer Design. Primers and probes for human UCHL1 gene and housekeeping ACTB, as well as universal probe library (UPL) hydrolysis probes, were designed using ProbeFinder 2.48 (Roche, Germany) software, UCHL-1 forward: CCTGAAGACAGAGCAAAATGC and reverse: TGTCATCTACCCGACATTGG (Genomed, Poland) with detection system of UPL 27 probe amplified 95 nucleotide region at the border of constitutively expressed exons (4/5). Reference sequence for UCHL1 gene was NM_004181.4 (NCBI, USA).

3.6. Relative Gene Expression by Real-Time PCR (RT-PCR). The RT-PCR reaction was performed in Roche LightCycler 480 II Thermocycler (Roche, Germany) in a volume of $10 \mu \mathrm{l}$. The reaction volume consisted of $5 \mu \mathrm{l}$ of LightCycler UPL ProbeMaster (Roche, Germany), 0,5 $\mu \mathrm{M}$ forward and reverse primers, and $0.2 \mu \mathrm{M}$ UPL probe 27 and $1 \mu \mathrm{l} \mathrm{cDNA}$. The reaction conditions were as follows: preincubation at $95^{\circ} \mathrm{C}$ by $10 \mathrm{~min}$., 50 cycles of amplification step: $95^{\circ} \mathrm{C}$ by $15 \mathrm{~s}$ for denaturation, $58^{\circ} \mathrm{C}$ by $1 \mathrm{~min}$. for elongation step, and $10 \mathrm{~s}$ at $72^{\circ} \mathrm{C}$ for detection and next the plate was cooled at $40^{\circ} \mathrm{C}$ for $10 \mathrm{~s}$. The relative gene expression was presented using $2^{-\Delta \Delta \mathrm{Ct}}$ method.

3.7. Immunofluorescence. The frozen skin sections and $\mathrm{HaCaT}$ fixed cells underwent the same immunofluorescence protocol with additional preincubation step for skin frozen sections with incubation for 10 minutes in solution of $5 \%$ acetic acid (POCH, Poland) in PBS and blocking step for cells and sections in blocking solution (BS): $3 \%$ bovine serum albumin (BSA) (LabEmpire, Poland), 5\% normal donkey serum (Jackson Immunoresearch, USA), 0.05\% Tween 20 (Sigma-Aldrich, Germany), and 0.01\% Triton-X100 (SigmaAldrich, Germany) in PBS with incubation time of 1 hour at $4^{\circ} \mathrm{C}$. After blocking step and 3x PBS rinsing, cells and sections were incubated with primary mouse anti-human PGP 9.5 antibody (13C4 clone, Ultraclone, United Kingdom) diluted in PBS (1:500) for 24 hours at $4^{\circ} \mathrm{C}$ temperature. Negative control was prepared by omitting the primary antibody and IgG-Alexa Fluor 488 isotype control (BD Bioscience, USA). Next day, sections were rinsed three times in PBS for $5 \mathrm{~min}$. and incubated for 2 hours at RT in dark conditions with the secondary donkey anti-mouse Alexa Fluor $546 \mathrm{H}+\mathrm{L}$ (A-10036, Invitrogen, USA) antibody in PBS (dilution 1:500). In turn, donkey anti-mouse Alexa Fluor $488 \mathrm{H}+\mathrm{L}$ (A-21202, Invitrogen, USA) was used for detection of PGP 9.5 in HaCaT cultures with the same conditions. The immunofluorescence mounting medium with DAPI (Invitrogen, USA) was used for nucleus counterstaining.

3.8. Western-Blot. Skin biopsies were homogenized in $400 \mu \mathrm{l}$ of cold PBS using electric tissues homogenizer (ProScientific, USA). After disruption, $400 \mu \mathrm{l}$ of $2 \mathrm{X}$ Radioimmunoprecipitation Assay Buffer (RIPA, $50 \mathrm{mMTris}-\mathrm{HCl}, 150 \mathrm{mMNaCl}$, $0.1 \%$ SDS, $1 \%$ NP-40, 0.5\% DOC, and $\mathrm{pH}=8.0$ ) was added with proteinase cocktail inhibitor (Sigma-Aldrich, Germany). Next, probes underwent centrifugation $\left(15 \mathrm{~min} .10 \times 10^{3} \mathrm{~g}, 4^{\circ} \mathrm{C}\right)$ and supernatants were collected. The protein concentration was determined using a BCA Protein Assay Kit (Pierce, IL, USA,). The $40 \mu \mathrm{g}$ of protein lysates was mixed with Laemmli's loading buffer $(4: 1)$ and denatured by incubation at $95^{\circ} \mathrm{C}$ for $5 \mathrm{~min}$. and immediately transferred on ice. As reference material, the $3 \mu \mathrm{g}$ of normal human hippocampus (Clontech Laboratories, USA) was used. The $12 \%$ SDS-PAGE gel electrophoresis was performed and protein transfer was verified by Coomassie Brilliant Blue (Sigma, USA) staining. Next, semidry electrotransfer from gels to PVDF and Whatman membranes $(0.45 \mu \mathrm{m}$, Millipore, USA) was performed for one hour (Bio-Rad, USA). In the next step, blotted membranes were hydrated by rinsing 3 times for $5 \mathrm{~min}$. in solution of Tris-buffered saline with $0.05 \%$ Tween 20 (TBST) and incubated with $1 \%$ casein solution in TBST $(0.1 \%$ Tween20 ) at RT for 1 hour. Next, the primary mouse anti-human PGP 9.5 (1:10000, Utraclone, 13C4 clone), the same antibody used for immunofluorescence, was diluted in TBST with $1 \%$ casein and incubated overnight at $4^{\circ} \mathrm{C}$. Rabbit polyclonal anti-human $\beta$-actin $(1 \mu \mathrm{g} / \mathrm{ml}, 1: 2000$, Cat. num.: SC-7210, Santa Cruz Biotechnology, USA) was used as reference. Next day, membranes were incubated with secondary antibodies conjugated with horseradish peroxidase-HRP diluted in blocking solution. Polyclonal goat anti-mouse-HRP (1:1000, Cat. num. P0447, Dako, Denmark) was used for PGP 9.5, while polyclonal goat anti-rabbit-HRP (1:1000, Cat. num.: P0448, Dako, Denmark) was used for $\beta$-actin detection. The

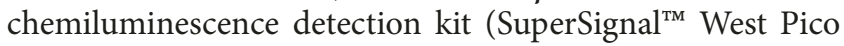
Chemiluminescent, Cat. num.: PI-34078, Thermo Scientific, USA) was used for bands visualization. 
3.9. Image Analysis. For frozen skin sections, immunofluorescence detection was carried out using the fluorescence microscope (Zeiss, Germany) with constant exposure conditions (1000 ms), microscope camera settings (Zeiss, Germany) at Zeiss Vision Image (ZVI) format using AxioVision software (Zeiss, Germany). In turn, positive immunofluorescence in HaCaT cells was detected and analyzed using the scanning confocal microscopy (Zeiss, USA). All fluorescence ZVI images were converted into the 8-bit RGB TIFF image files and transferred to the Fiji software (Fiji, ImageJ, National Institute of Health, Bethesda, USA).

3.10. Statistical Analysis. All statistical analyses were performed using Statistica 12.0 software (Dell Software, USA). Differences between samples from compared patient groups were verified using Mann-Whitney $U$ test. Correlation data were analyzed by Spearman rank correlations test. Values of $p<0.05$ were considered statistically significant.

3.11. Data Presentation. The presented graphs were performed using GraphPad Prism 5.0 (GraphPad Software Inc., La Jolla, CA, USA), while figures were prepared by LibreOffice 5.0 Software (The Document Foundation, Germany).

\section{Results}

Results of our investigations demonstrate cutaneous expression of UCHL1/PGP 9.5: UCHL1 gene transcript, as well as PGP 9.5 protein expression in total skin biopsy samples. We detect PGP 9.5 by basal (BKs) and suprabasal (SBKs) epidermal keratinocytes as well as DRG axonal nerve terminals and dermal nerve fibers, respectively (graphs and images presented below). Furthermore, barely but stable expression within immortalized $\mathrm{HaCaT}$ cells was also demonstrated (Figure 1: (a1)-(a3), (b), and (c)). All reagents used for detection of UCHL1 transcript and PGP 9.5 protein were confirmed in the human brain reference material (Figure 1(c)).

4.1. PGP 9.5 Distribution and Expression in the Skin of Controls and Psoriasis Patients. The PGP 9.5 somatosensory system was detectable in the skin of both healthy and psoriatic subjects. In normal epidermis, PGP 9.5 signal was present in axonal nerve terminals and dermal nerve fibers. The single nerve terminals penetrating all epidermal layers were detected only occasionally in stratum spinosum or granulosum. We observed single nerve terminals in normal dermis, which in some subjects crossed dermal-epidermal border. A perivascular PGP 9.5 expression was also observed in dermal microvascular network, sweet glands and dermal papillae (Figure 2(a)). Furthermore, cytoplasmic expression of PGP 9.5 was demonstrated in normal BKs and considered as single or group arranged cells. Normal SBKs demonstrate almost complete absence of PGP 9.5 with some barely and heterogeneous expression via single epidermal keratinocytes (Figure 2(a)). The nonlesional epidermis showed numerous nerve terminals, while some of them even reached stratum corneum, with strong expression of PGP 9.5 within BKs (Figures 2(b) and 2(d)). In turn, PGP 9.5 expression in psoriatic lesions was more diversified and demonstrated gradual expression patterns. The PGP 9.5 expression was observed in BKs with free PGP 9.5 epidermal nerve terminals or without detectable expression within BKs but with tendency to turn over from weak PGP 9.5 nerve ending expression to strong PGP 9.5 expression by SBKs. Nerve terminals in skin lesions seemed to be more elongated in comparison to nonlesional skin and healthy individuals (Figures 2(c) and 2(e)).

4.2. UCHL1 Gene Expression in the Skin Biopsies. The expression of $U C H L 1$ gene in skin biopsies of psoriasis patients was downregulated in comparison to group of healthy individuals (Figure 3(A)). UCHL1 expression in nonlesional psoriatic skin (mean: 0.002; SD: $\pm 0.0008, \mathrm{p}<0.001$ ) was significantly lower in comparison to control group (mean: 0.008; SD: $\pm 0.007)$. The psoriasis patients without itch in nonlesional (mean: 0.001, SD: \pm 0.00065 ) and lesional skin (mean: 0.002, SD: \pm 0.001$)$ did not demonstrate significant differences of UCHL1 expression compared with control group (mean: 0.008 , SD: \pm 0.007$)$. However, nonlesional skin without itch (mean: 0.00098, SD: \pm 0.0007$)$ demonstrated significantly lower UCHL1 expression in comparison to nonlesional skin with itch (mean: 0.0019, SD: $\pm 0.0007, \mathrm{p}=0.04$ ). While skin lesions with itch (mean: 0.0018, SD: \pm 0.0035 ) demonstrate significantly decreased UCHL1 expression compared to control group (mean: $0.008, \mathrm{SD}: \pm 0.007, \mathrm{p}=0.02$ ), such differences were not observed between psoriatic lesions without itch (mean: 0.00174, SD: \pm 0.0016 ) and normal skin (mean: 0.008; SD: $\pm 0.007, \mathrm{p}=0.15$ ) (Figure 3(C)).

4.3. Density of PGP 9.5 Epidermal Nerve Terminals and Dermal Nerve Fibers. The numbers of epidermal PGP 9.5 axonal nerve terminals (mean: 4.5 , SD: $\pm 1.47 ; \mathrm{p}<0.001$ ) and dermal nerve fibers (mean: 2.53 , SD: $\pm 1.44 ; \mathrm{p}<0.01$ ) were significantly increased in nonlesional skin of patients with psoriasis compared to epidermis (mean: $2.6, \mathrm{SD}: \pm 1.39$; $\mathrm{p}<0.001$ ) and dermis (mean: $1.21, \mathrm{SD}: \pm 0.53$; $\mathrm{p}<0.01$ ) of healthy individuals (Figure 3(B)). In relation to psoriatic skin lesions, the number of epidermal PGP 9.5-positive axonal nerve terminals (mean: 4.5 SD: $\pm 4.5 ; \mathrm{p}=0.07$ ) and dermal fibers (mean: 2.1, SD: $\pm 1.8 ; \mathrm{p}=0.04)$ seems to be higher than their epidermal (mean: 2.6, S.D.: \pm 1.4 ) and dermal (mean: 1.2, SD: \pm 0.5 ) control counterparts (Figure 3(A)). In relation to itch, we observed significantly higher number of epidermal nerve terminals (mean: 4.93, SD: $\pm 1.21, \mathrm{p}<0.001$ ) and dermal nerve fibers (mean: 2.94, SD: \pm 1.21 ) in nonlesional skin with itch compared to healthy subjects (mean: $1.21, \mathrm{SD}: \pm 0.53 ; \mathrm{p}=0.001$ ) (Figures 2(a) and 2(b); Figure 3(D)). We also observed significant differences between nonlesional epidermis with itch (mean: 4.93, SD: \pm 1.21 ) compared to nonlesional skin without itch (mean: 3.50 , SD: $\pm 1.64 ; \mathrm{p}<0.05$ ). In turn, itchy nonlesional dermis (mean: 2.93, SD: \pm 1.42 ) seems to have more fibers compared to that without itch (mean: 1.58, SD: \pm 1.04 ), but the difference did not reach the statistical significance $(\mathrm{p}=0.07)$ (Figures 2(b) and 2(d)). Moreover, epidermis (mean: 5.86, SD: \pm 4.74 ) of itchy lesions, similarly to dermis (mean: 2.56, SD: \pm 1.83 ), had higher distribution of axonal nerve terminals and fibers compared to epidermis (mean: 1.50 , SD: $\pm 1.05 ; \mathrm{p}=0.04$ ) and dermis without itch (mean: 1.03 , SD: \pm 1.02 ; $\mathrm{p}=0.07$ ); however, statistical significance was 

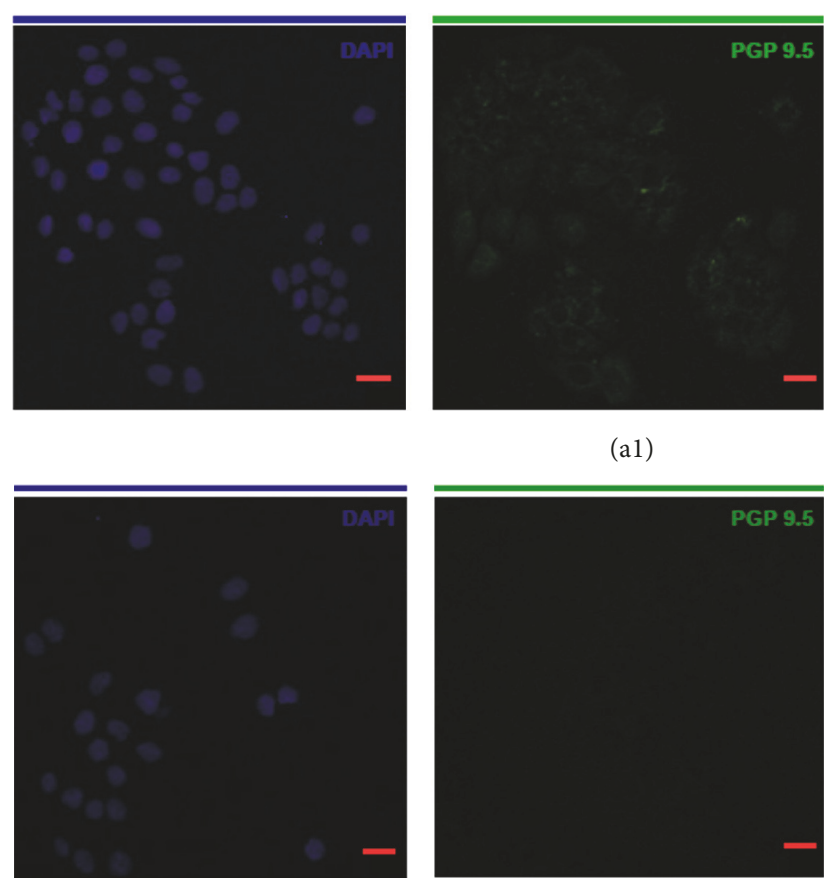

(a1)

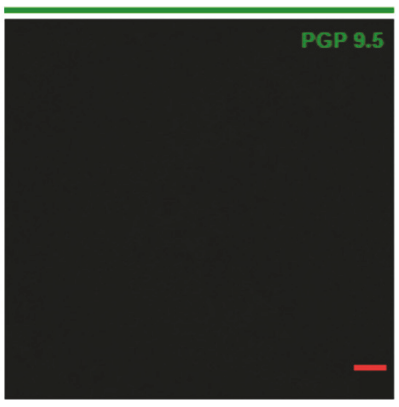

(a2)
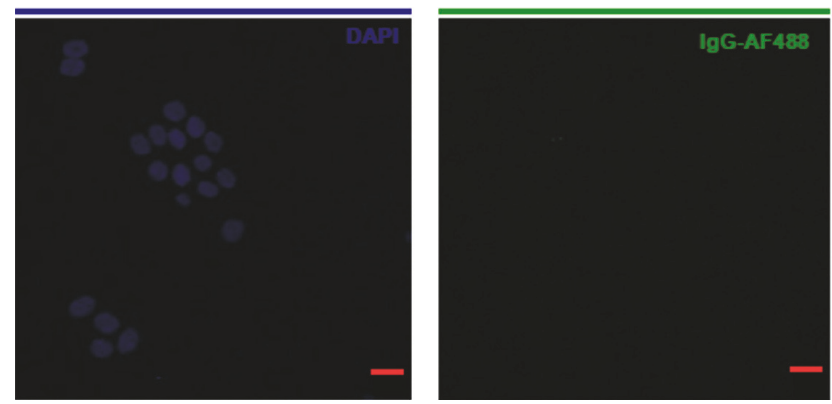

(a3)
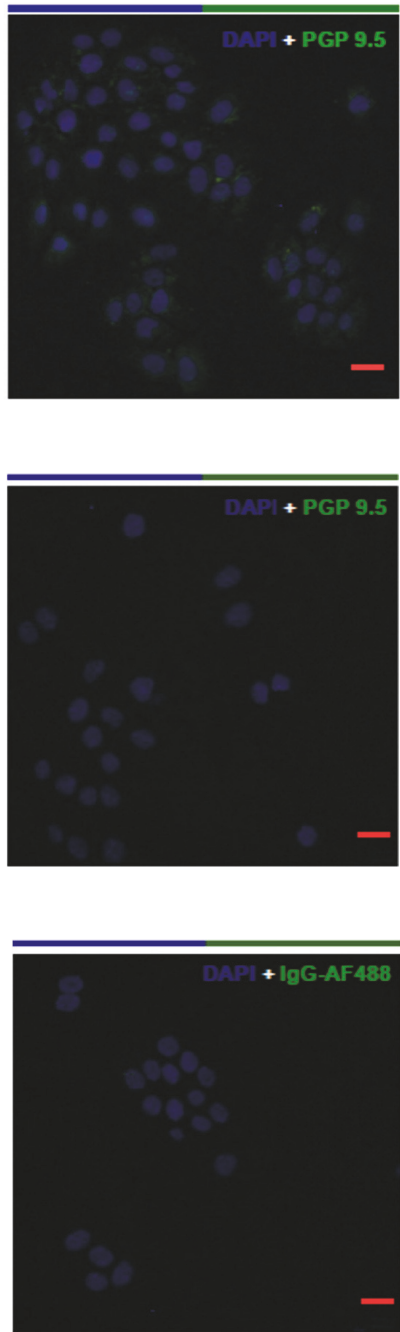

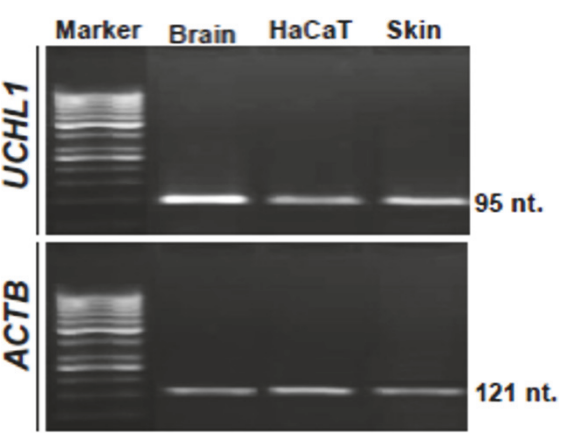

(b)

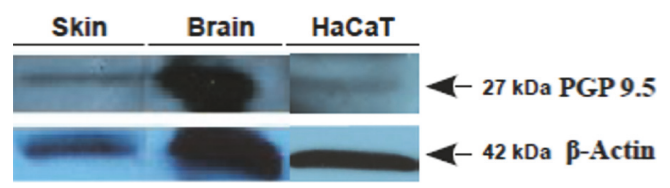

(c)

FIgURE 1: Expression of UCHL1 gene and PGP 9.5 protein in skin punch biopsies, HaCaT keratinocyte cell line, and brain reference material. Confocal scanning microcopy using HaCaT keratinocyte cell line presents images of cytoplasmatic expression of PGP 9.5 protein (a1). Furthermore, omission of primary antibody against human PGP 9.5 (a2) as well as IgG-AF488 isotype control does not result in any specific signal (a3). Scale bars represent $20 \mu \mathrm{m}$. UCHL1 expression at mRNA level was observed in HaCaT cell line, normal skin, and was confirmed in brain reference material. Forward and reverse primers amplificate 95nt sequence at the border of exons 3 and 4 of $U C H L 1$ gene detected using FAM-labeled UPL 27 probe. ACTB was used as housekeeping gene using VIC-labeled probe and amplificate 121nt fragment. All amplified material was visualized using gel electrophoresis (b). Protein expression was verified using Western-blot with the same mouse anti-human PGP 9.5 antibody and gave specific $27 \mathrm{kDa}$ band in the skin, brain, and HaCaT cell line, respectively (c). 


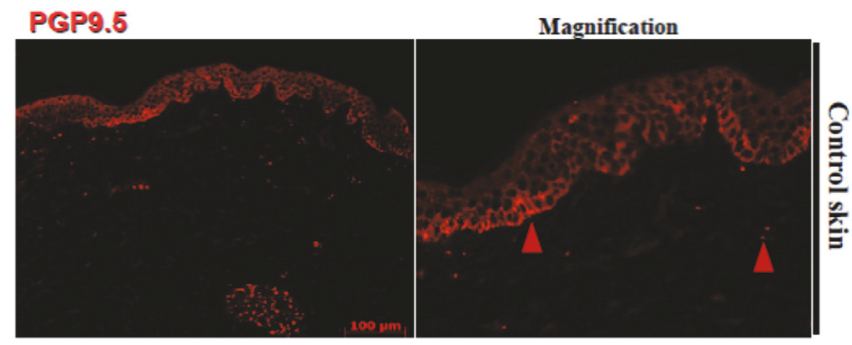

(a)

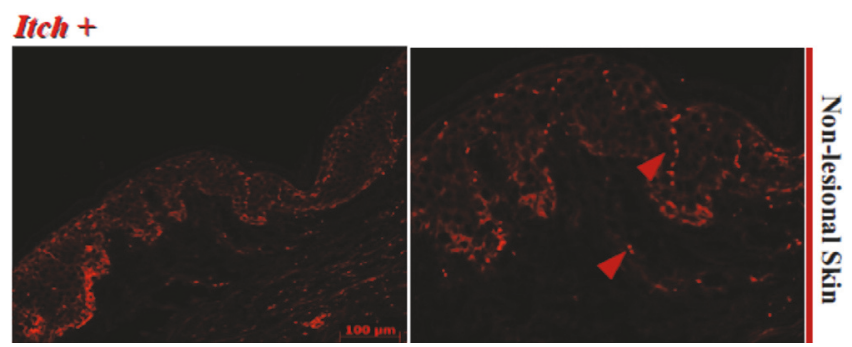

(b)

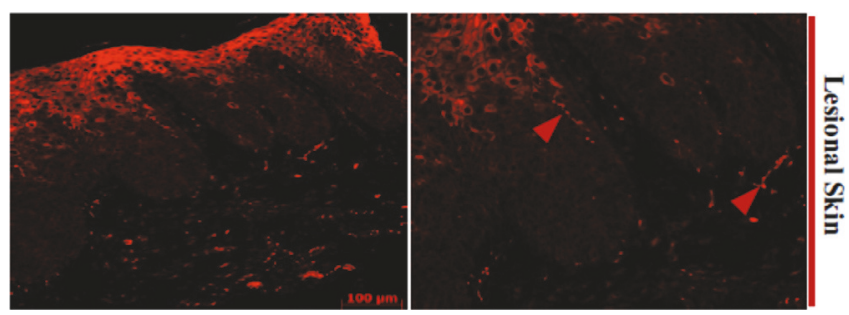

(c)

Iich -
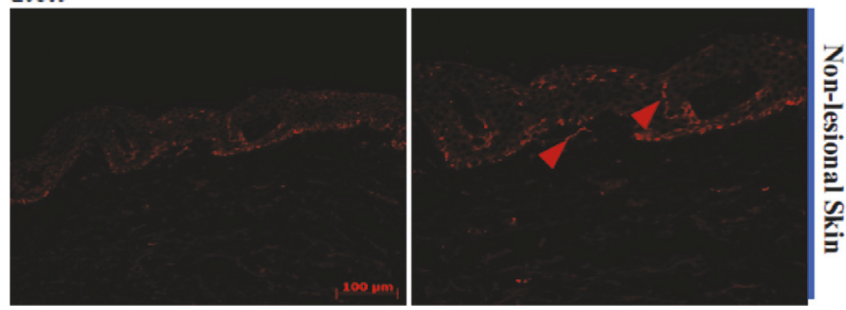

(d)

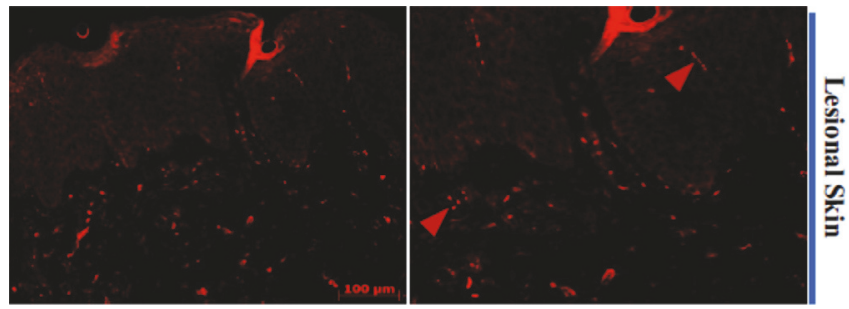

(e)

FIGURE 2: PGP 9.5 distribution and expression in axonal nerve terminals and dermal nerve fibers in skin samples of healthy individuals and nonlesional and lesional skin of psoriasis with or without itch. PGP 9.5 of epidermal axonal nerve terminals and dermal fibers in normal healthy skin analyzed by indirect immunofluorescence staining on frozen skin sections (a). The nonlesional (b) and lesional skin (c) with itch $(I t c h+)$, in relation to nonlesional (d) and lesional skin (e) without itch (Itch-) from psoriasis patients. Axonal nerve terminal and dermal nerve fibers marked with red arrows. Scale bars represent $100 \mu \mathrm{m}$. 


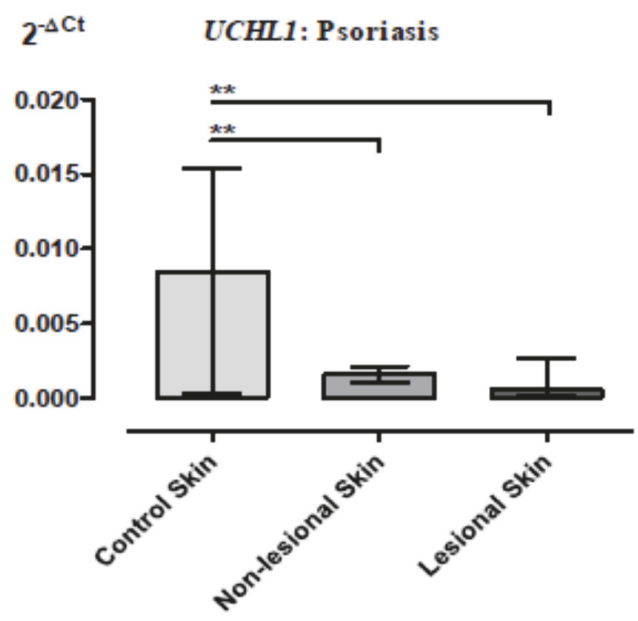

(A)

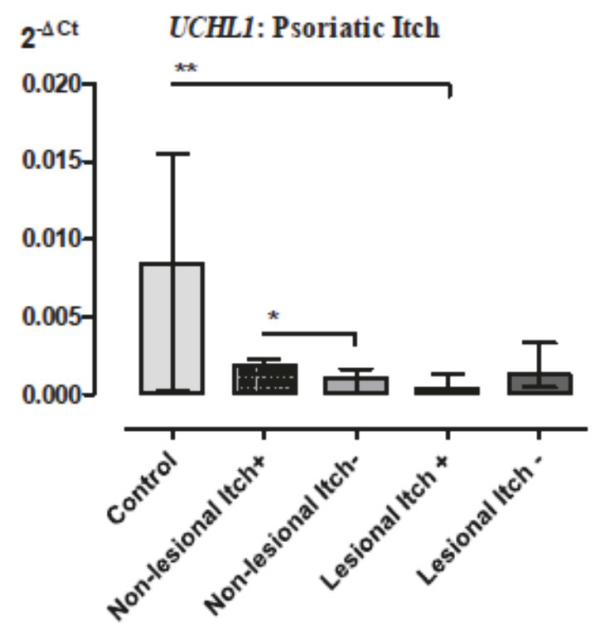

(C)

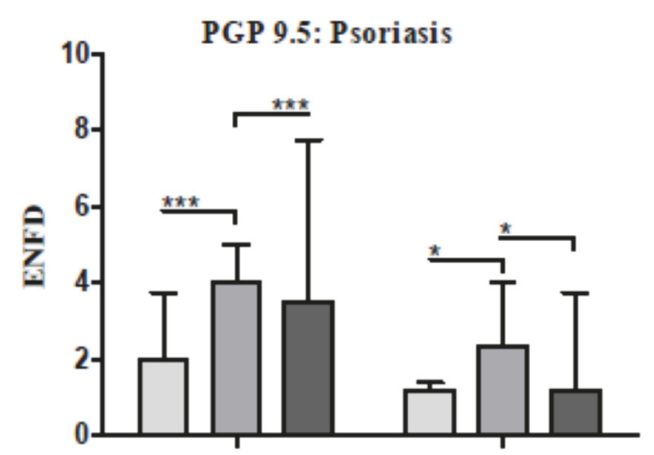

a.

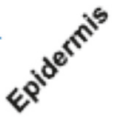

b.

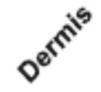

(B)

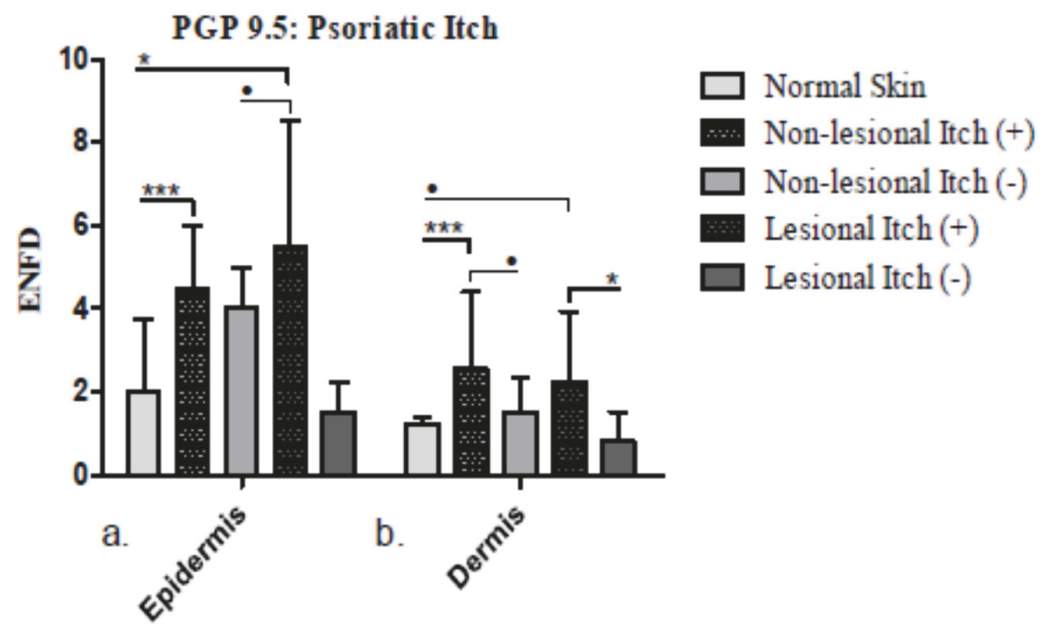

(D)

FIGURE 3: Graphs summarizing results of relative UCHL1 gene expression and PGP 9.5 expression within epidermal axonal nerve terminals and dermal fibers in the skin of healthy control and psoriatic patients with division into with (Itch+) and without (Itch-) itch. The relative expression of UCHL1 gene in psoriasis (A) and epidermal nerve fiber density (ENFD) of PGP 9.5 axonal nerve terminals and dermal nerve fibers in nonlesional and lesional skin of psoriasis (B) along with the division into with (itch+) and without (itch-) itch $((\mathrm{C}),(\mathrm{D}))$.

achieved only regarding the epidermis (Figures 2(c) and 2(e); Figure 3(B)). Furthermore, we observed the positive correlation between VAS score and both PGP 9.5 epidermal axonal nerve terminals $(\mathrm{r}=0.47 ; \mathrm{p}<0.05)$ and number of dermal nerve fibers $(r=0.42 ; \mathrm{p}<0.05)$ in lesional psoriatic skin (data not shown). In addition, reverse correlation between disease severity (PASI score) and both PGP 9.5 expression in lesional epidermis $(r=-0.51 ; p<0.05)$ and dermis $(r=-0.45$; $\mathrm{p}<0.05)$ was also observed.

4.4. PGP 9.5 Basal (BKs) and Suprabasal (SBKs) Keratinocyte Expression. In general, we demonstrated significantly higher cytoplasmic expression of PGP 9.5 protein by basal (BKs) as well as suprabasal keratinocytes (SBKs) than controls (Figure 4). The BKs of nonlesional skin demonstrated significantly higher expression of PGP 9.5 protein (mean: 35.1,
SD: $\pm 22.6 ; \mathrm{p}=0.001$ ), and SBKs did (mean: 21.6, SD: \pm 7.0 : $\mathrm{p}=0.0001$ ) in comparison to BKs (mean: 17.2, SD: \pm 3.6 ) and SBKs (mean: 14.3, SD: \pm 2.2 ) of control group (Figures 4(a), 4(b), and 4(d); Figures 5(a) and 5(b)). Furthermore, nonlesional PGP 9.5-expressing SBKs positively correlated with VAS (SBKs: $\mathrm{r}=0.49, \mathrm{p}=0.02$ ), while, regarding PGP 9.5expressing BKs, no significant correlation with VAS was noted (BKs: $r=0.35, p=0.13$ ). In lesional skin, significant discrepancies of PGP 9.5 expression concerned only SBKs (mean: 32.5, SD: \pm 17.2 , p<0.001) when compared to controls (mean: 14.3, SD: \pm 2.2 ) (Figure 5(b)), while psoriatic BKs (mean: 19.2, SD: $\pm 14.3 ; \mathrm{p}=0.54$ ) and controls (mean: 14.3, SD: \pm 2.2 ) demonstrated comparable PGP 9.5 expression (Figures 4(a), 4(c), and 4(e); Figure 5(a)). PGP 9.5 expressing SBKs in psoriatic lesions positively correlated with itch regarding VAS score (SBKs: $\mathrm{r}=0.64, \mathrm{p}=0.002$ ). The differences were also 

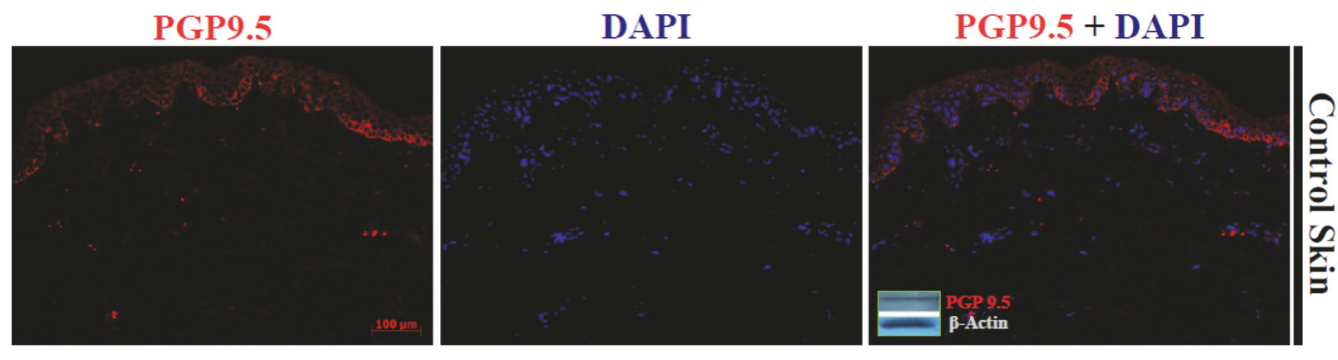

(a)
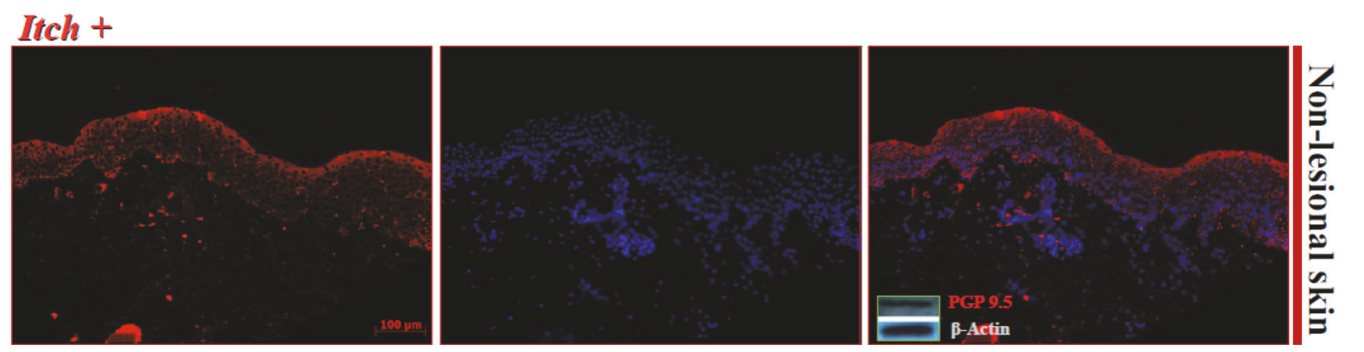

(b)
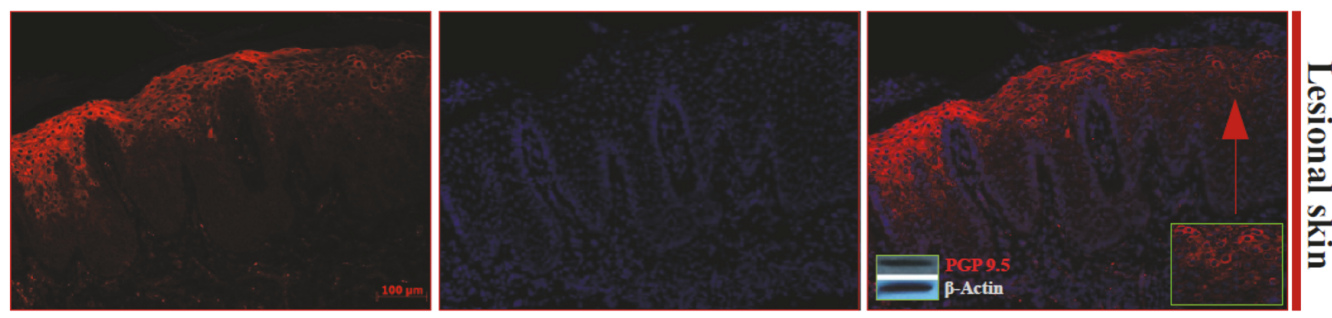

(c)
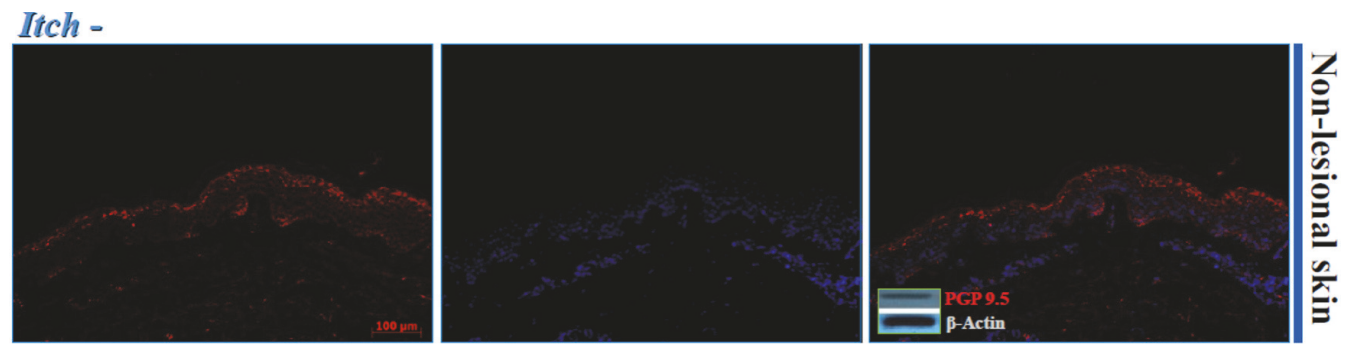

(d)
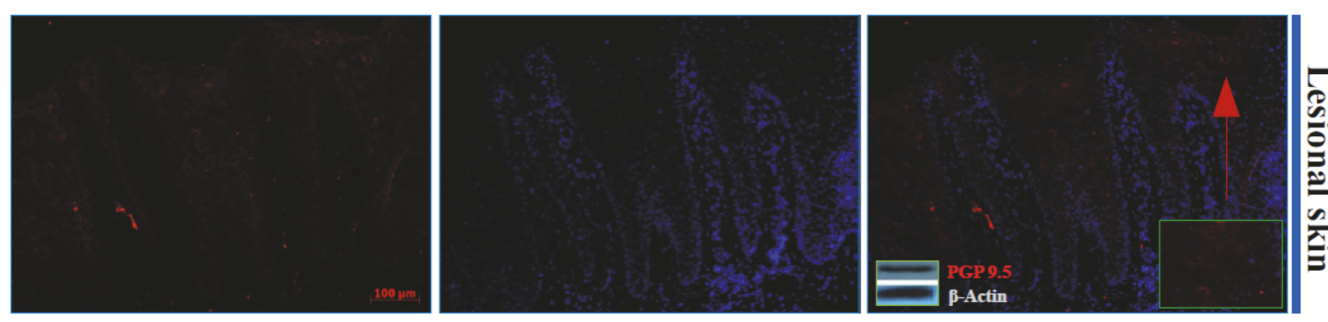

(e)

FIGURE 4: Expression of PGP 9.5 by epidermal keratinocytes in control skin and nonlesional and lesional skin with (Itch+) and without (Itch-) itch. Immunofluorescence images of epidermal expression of PGP 9.5 in control healthy skin was found mostly in basal keratinocytes (BKs) with weak detection by suprabasal keratinocytes (SBKs) (a). Further images represent BKs and SBKs PGP 9.5 expression in nonlesional and lesional samples with ((b), (c); Itch+) and without ((d), (e); Itch-) itch. Inserts: western-blot analysis of PGP 9.5 expression of the representative skin samples of two controls as well as two patients with (Itch+) and two without (Itch-) itch, respectively. Representative bands of PGP $9.5(27 \mathrm{kDa})$ and $\beta$-actin $(42 \mathrm{kDa})$ endogenous control are listed in the lower right corners of merged images. 
PGP 9.5: Basale Keratinocytes (BKs)

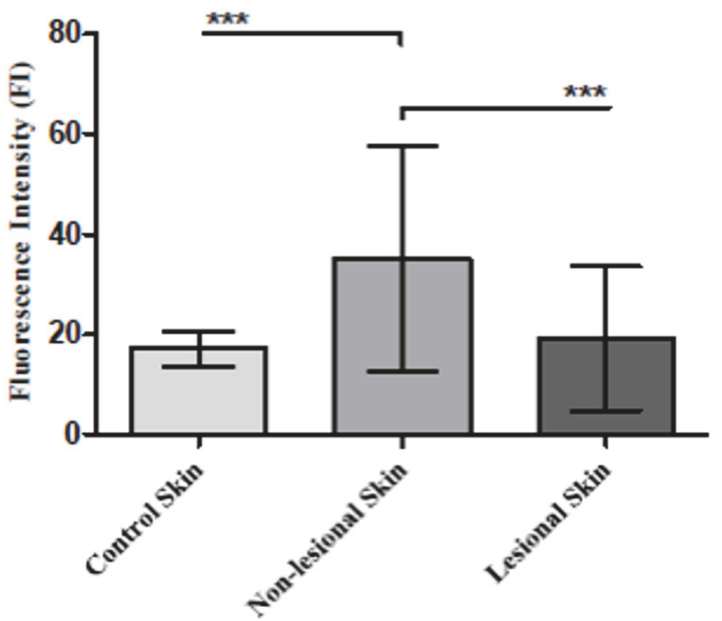

(a)

PGP 9.5: Suprabasal Keratinocytes (SBKs)

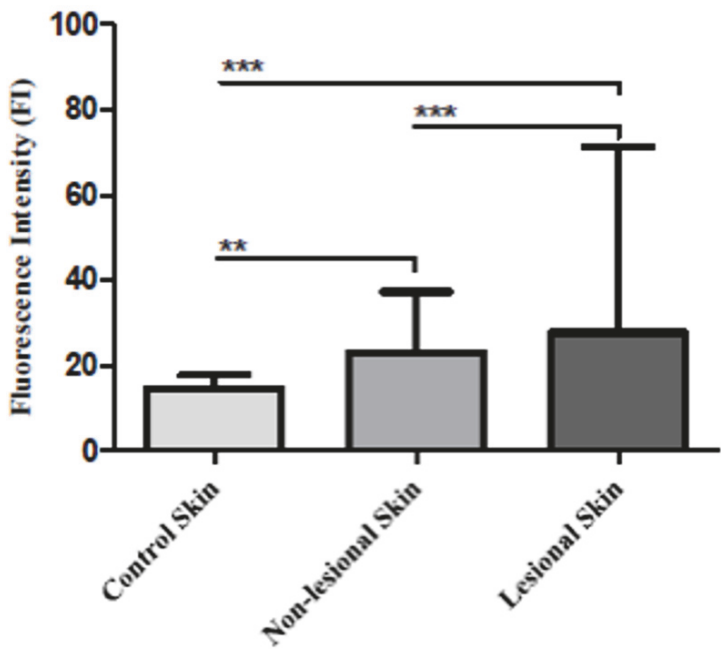

(c)
PGP 9.5: Basal Keratinocytes (BKs)

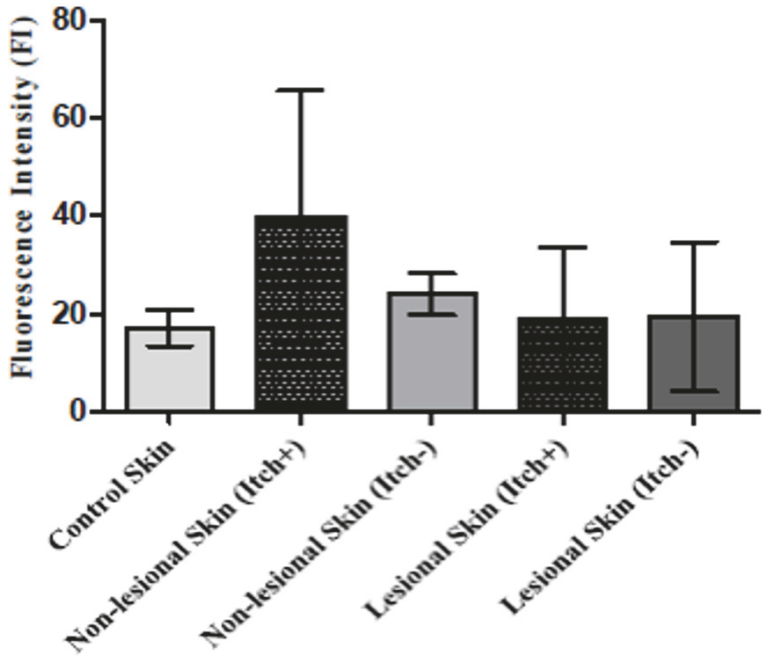

(b)

PGP 9.5: Suprabasal Keratinocytes (SBKs)

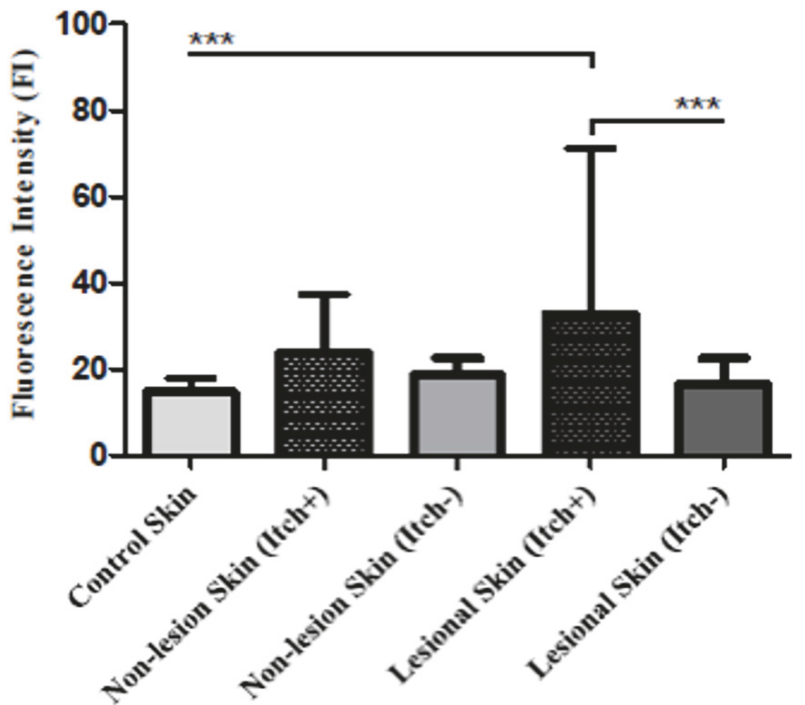

(d)

FIGURE 5: Graphs summarizing results of semiquantitative fluorescence intensity (FI) of PGP 9.5 expression by BKs and SBKs in the skin of healthy controls and psoriatic patients with (Itch-) and without (Itch-) itch. The semiquantitative fluorescence intensity of BKs and SBKs in healthy control persons and nonlesional and lesional skin of psoriasis patients ((a), (c)) with (itch+) and without (itch-) itch ((b), (d)).

observed between nonlesional BKs (mean: 26.1, SD: \pm 18.4 ; $\mathrm{p}<0.001$ ) compared to BKs of psoriatic lesions (mean: 18.2, SD. \pm 10.38 ) (Figures 4(b), 4(c), 4(d), and 4(e); Figure 5(a)). SBKs of cutaneous lesions (mean: 23.37, SD: $\pm 15.23 ; \mathrm{p}=0.007$ ) expressed PGP 9.5 at significantly higher level compared to nonlesional skin (mean: 17.93, SD: \pm 6.34 ) counterparts (Figure 5(b); FI: Figures 4(c), 4(e), 4(b), and 4(d)). Furthermore, only SBKs of itchy skin lesions (mean: 38.91 , SD: \pm 16.72 ; $\mathrm{p}=0.006$ ) presented significantly increased expression of PGP
9.5 in comparison to lesional skin without itch (mean: 17.42, SD: \pm 3.88 ) (Figures 4(c) and 4(e); Figure 5(d)).

\section{Discussion}

Expression of UCHL1/PGP 9.5 system is observed in cells with neuro-immune-endocrine activity and DRG neurons, in which terminals indirectly interplay with epidermal keratinocytes and are fundamental organizers of the cutaneous 
somatosensory complex $[25,26]$. Experimental sciatic nerve injury in animals demonstrated rapid downregulation of UCHL1/PGP 9.5 expression by nerve terminals, whereas its amounts became compensated mainly by Langerhans cells (LCs) and Merkel cells (MCs), respectively [15, 25, 27]. However, several studies indicated that expression of PGP 9.5 may also be considered normal epidermal keratinocytes and dermal fibroblasts [28]. In turn, rapid induction of UCHL1/PGP 9.5 by cells of nonneuronal origin, like keratinocytes, have been discussed and multiple scenarios were proposed [28, 29]. The keratinocytes engagement in itch-mediated signals was proved in a number of studies using in vitro assays [26, 30]. In turn, studies from clinics demonstrated involvement of nervous system in psoriasis, and animal models confirmed these relations $[25,31-33]$. In context of current studies, reverse correlation between higher PGP 9.5 protein and its lower UCHL1 gene expression was observed, with emphasis on the lowest UCHL1 in itchy lesions. Thus, essential question has arisen of whether decreased UCHL1 expression in psoriasis might be linked with impaired function of neuroimmune-cutaneous milieu. LCs impaired density is usually observed in vivo in pain-related syndromes. Keratinocytes and PGP 9.5 ${ }^{+}$LCs closely cooperate via direct contact with itch-mediating PGP 9.5 ${ }^{+}, \mathrm{CGRP}^{+}$neurons [34]. CGRP is a well-known cell-immunity modifier, which abolishes LCs antigen presentation. Its increased amounts were noted to be released from nerve terminals in psoriasis $[34,35]$. Opposite results were obtained by Sebastian et al., who demonstrated that, in electrically induced wound injury model, expression of UCHL1 gene and PGP 9.5 was significantly increased at nerve terminals, but also in MCs together with other commonly applied neuronal markers [36]. Keratinocytes in psoriatic lesions are neuroinflammatory cells and induced expression of other neurorelated proteins may reflect their injured conditions [33]. Furthermore, PGP 9.5 positive branches of DRG neurons are significantly increasing in the presence of infiltrating cells like eosinophils, both in vitro and in vivo in atopic dermatitis, and this mechanism should not be negated in psoriasis [35]. In psoriasis, number of proinflammatory mediators, such as prostaglandins, is elevated, whereas their reactive lipid compounds contribute to molecular modification of covalently bounded cysteine residues in structure of PGP 9.5, e.g., in brain studies [37]. Whether inducible expression of UCHL1/PGP 9.5 system in nonneuronal cells like keratinocytes may be a sign of appearing molecular dysfunction and participate in transmission of subjective sensations still needs to be confirmed. However, Johansson et al. indicated that single keratinocytes in suprabasal compartments of normal epidermis exhibit PGP 9.5 expression $[28,29]$. Inducible expression of UCHL1 was seen in vitro in primary outgrowing keratinocytes arising from psoriasis patients, while in microdissected skin it was only slightly elevated [38]. In turn, Eiding et al. studies presented that UCHL1 gene was significantly increased in nonlesional and lesional keratinocytes derived from epidermis of psoriasis patients, especially in those subjected exposed to UVB irradiation [32]. The series of further studies demonstrated that UCHL1/PGP 9.5 system may regulate melanogenesis dependent on agouti signal protein (ASIP,
ASP in mice). ASIP is a naturally widely expressed tissue inhibitor for melanocortin-1 receptor (MC1R) and is located in so-called "ITCHy" locus. The locus contains principal proteins and transcription factors responsible for skin development. Moreover its name arises from being situated there HECT-ITCH ligase E3 enzyme also belonging to ubiquitin system. Its genetic knockdown results in development of nonagouti-lethal $18 \mathrm{H}$ mice with spectrum of immune dysfunction and main behavior of uncontrolled scratching of the skin $[39,40]$ Melanocyte cell line treated by ASP recombinant demonstrates rapid upregulation of UCHL1, together with genes responsible for axonal guidance and neuronal development [41]. Moreover, Young Seo and coworkers showed that overexpression of UCHL1/PGP 9.5 by melanocytes resulted in microphthalmia-associated transcription factor (MTIF) ubiquitination and degradation, with dramatic reduction of pigmentation [42]. MITF consists of critical protein responsible for melanocytes development and melanogenesis, so it is possible that phototherapy-induced release of UCHL1/PGP 9.5 via melanocytes may enhance its horizontal transfer to the neighboring keratinocytes using exosome-dependent pathway [43]. Melanocytes in the epidermis are located in the basal layer, while their prominent and long dendrites penetrate to the upper epidermal compartments participating in melanin transfer to the surrounding keratinocytes [44]. In psoriasis, melanocytes are elevated; however, MITF expression was noted to be comparable to controls [45]. The attention should be also focused to other studies where model of experimental skin injury demonstrated participation of PGP 9.5 with other axonal transport factors in synaptic cargo vesicles from DRG bodies to the periphery [46]. Thus, whether stable expression of PGP 9.5 protein within human epidermal keratinocytes is MITF-independent and whether intracellular communications are engaged should be further elucidated.

The epigenetic cytosine-phosphate-guanine ( $\mathrm{CpG})$ DNA hypermethylation of UCHL1/PGP 9.5 promoter is a wellknown mechanism responsible for partial or complete silencing of the expression within tissues and was well demonstrated in melanoma cell lines and patients, respectively [47]. Several studies using different spectrum of molecular techniques underlined infections, oxidative stress, or immune-mediated disorders as possible factors down- or upregulating UCHL1/PGP 9.5 in PNS cells as well as in nonneuronal cells $[10,48,49]$. Using siRNA technique, UCHL1/PGP 9.5-expressing cell lines showed more than 200 downregulated and comparable number of upregulated genes engaged in most cellular processes, also those responsible for molecular background of psoriasis [50]. So, molecular mechanisms of protein ubiquitination seem to be deregulated in inflammatory-associated diseases and other ubiquitinrelated enzymes participating in signaling pathways are analyzed to better explain their roles in psoriasis disease [51]. Furthermore, new findings connect neurodegenerative symptoms and neuropathic pain with activation of ubiquitination pathways; however, sensations, such as itch, are still poorly explained in that context [11]. Increased numbers of PGP 9.5 axonal nerve terminals and dermal nerve fibers in the skin are considered as the main itch transmitting factor and 
our data confirmed association of altered PGP 9.5 neuroepidermal profile with itch. Nakamura et al. have demonstrated that psoriasis patients have higher number of PGP 9.5 nerve terminals in the epidermis which positively correlated with itch intensity [52]. Similar results were observed by Takamori et al.; however, in both studies analyses were only restricted to the diseased skin [53]. Johansson and colleagues indicated that PGP 9.5 nerve terminals are altered only in the skin with lesions, while nonlesional skin resembled the samples from controls. PGP 9.5 in psoriatic lesions were more frequently presented on the dermal-epidermal border and showed restricted pattern in epidermis, which is consistent with our observations [54]. In our study, the number of PGP 9.5 terminals in psoriatic lesions was comparable with healthy controls; however, those in epidermis and dermis seemed to be more elongated. In nonlesional skin, PGP 9.5 terminals were predominantly present in the epidermis. However, patients with itch had significantly higher number of PGP 9.5 nerve terminals in the epidermis and fibers in the dermis in comparison to the skin without itch and healthy control group. We also observed that number of PGP 9.5 axonal nerve terminals in the epidermis and dermis of lesional skin positively correlated with VAS score. Pergolizzi et al. demonstrated that epidermal PGP 9.5 nerve terminals in long-established skin lesions of psoriasis may undergo almost complete reduction. They postulate gradually occurring changes in the psoriatic lesions at initial phase of psoriasis with increased innervations and nerve projections through all epidermal layers, to almost complete reduction at advanced stages [55]. These results partially explained our observations and may depend on exacerbation phase of psoriasis [56]. The number of PGP 9.5 nerve terminals was also recorded as increased in samples taken from scalp of psoriatic patients; however, no relation with itch was found [57]. The participation of C-fibers in psoriatic itch was also confirmed by transcutaneous electrical nerve stimulation, where mean of somatosensory threshold level was significantly higher in itchy lesional skin [58]. Significantly altered PGP 9.5 nerve terminals were observed in group of stress exposed healthy volunteers. Acute psychoemotional stress conditions may increase the number of cutaneous PGP 9.5 nerve terminals. Thus, decreased neuropsychological conditions and skin neuroinflammation may also exacerbate PNS in psoriasis $[59,60]$.

\section{Conclusions}

PGP 9.5 profiling of axonal nerve terminals in skin samples of patients with psoriasis may constitute a useful method for assessment of itch. In turn, our studies for the first time indicated that increased itch-signal transmission might be a consequence of nonneuronal synapse dysfunctions. The interaction between nerve terminals penetrating epidermis and their terminations within neuro-immune-endocrine cells in psoriasis might consist one of the possible mechanisms. Thus, our findings suggest that disordered interplay between axonal transport and epidermal keratinocytes, as well as impaired ubiquitylation mechanisms, may constitute a new molecular player in itch transmitting pathways.

\section{Data Availability}

All data used to support the results of this study are included within the article.

\section{Conflicts of Interest}

The authors declare no conflicts of interest.

\section{Acknowledgments}

All investigations were financially supported by "Preludium" grant from the Polish National Science Center (UMO2011/01/N/NZ4/04946): "Gene and protein analysis of OPRM1/MOR and OPRK1/KOR opioid receptors expression and neurobiological marker of nerve terminals PGP 9.5 in skin of psoriasis patients with and without pruritus" realized in 2011-2013 years. Dmitry Nevozhay acknowledges support from the Ministry of Education and Science, Russian Federation, through Program 1326. The authors would like to extend special thanks to Dr. Mariusz Gajda for his excellent knowledge and technical support of peripheral nervous system and Dr. Marcin Hołysz for supporting all molecular biology procedures. Furthermore, they would like to specially thank Dr. Beata Nowakowska from Laboratory of Tissues Immunology, Ludwik Hirszfeld Institute of Immunology and Experimental Therapy Medical Centre, Polish Academy of Sciences, for her organizational support and incredible kindness.

\section{References}

[1] M. A. Lowes, M. Suárez-Fariñas, and J. G. Krueger, "Immunology of psoriasis," Annual Review of Immunology, vol. 32, pp. 227-255, 2014.

[2] T. Wang and C. Ma, "Peripheral nociceptors as immune sensors in the development of pain and itch," Advances in Experimental Medicine and Biology, vol. 904, pp. 77-85, 2016.

[3] R.-R. Ji, A. Chamessian, and Y.-Q. Zhang, "Pain regulation by non-neuronal cells and inflammation," Science, vol. 354, no. 6312, pp. 572-577, 2016.

[4] J. C. Szepietowski and A. Reich, "Itch in Psoriasis Management," Current Problems in Dermatology (Switzerland), vol. 50, pp. 102110, 2016.

[5] J. Jeffry, S. Kim, and Z.-F. Chen, "Itch signaling in the nervous system," Physiology Journal, vol. 26, no. 4, pp. 286-292, 2011.

[6] I. N. M. Day and R. J. Thompson, "UCHL1 (PGP 9.5): Neuronal biomarker and ubiquitin system protein," Progress in Neurobiology, vol. 90, no. 3, pp. 327-362, 2010.

[7] K. D. Wilkinson, K. M. Lee, S. Deshpande, P. Duerksen-Hughes, J. M. Boss, and J. Pohl, "The neuron-specific protein PGP 9.5 is a ubiquitin carboxyl-terminal hydrolase," Science, vol. 246, no. 4930, pp. 670-673, 1989.

[8] P. Bishop, D. Rocca, and J. M. Henley, "Ubiquitin C-Terminal hydrolase L1 (UCH-L1): Structure, distribution and roles in brain function and dysfunction," Biochemical Journal, vol. 473, no. 16, pp. 2453-2462, 2016.

[9] H. Osaka, Y.-L. Wang, K. Takada et al., "Ubiquitin carboxyterminal hydrolase L1 binds to and stabilizes monoubiquitin in neuron," Human Molecular Genetics, vol. 12, no. 16, pp. 19451958, 2003. 
[10] J. Coulombe, P. Gamage, M. T. Gray et al., "Loss of UCHL1 promotes age-related degenerative changes in the enteric nervous system," Frontiers in Aging Neuroscience, vol. 6, 2014.

[11] B. Genç, J. H. Jara, M. C. Schultz et al., "Absence of UCHL 1 function leads to selective motor neuropathy," Annals of Clinical and Translational Neurology, vol. 3, no. 5, pp. 331-345, 2016.

[12] M. Zhang, F. Cai, S. Zhang, S. Zhang, and W. Song, "Overexpression of ubiquitin carboxyl-terminal hydrolase L1 (UCHL1) delays Alzheimer's progression in vivo," Scientific Reports, vol. 4, p. 7298, 2014.

[13] R. Nejati, D. Kovacic, and A. Slominski, "Neuro-immuneendocrine functions of the skin: An overview," Expert Review of Dermatology, vol. 8, no. 6, pp. 581-583, 2013.

[14] O. Johansson, L. Wang, M. Hilliges, and Y. Liang, "Intraepidermal nerves in human skin: PGP 9.5 immunohistochemistry with special reference to the nerve density in skin from different body regions," Journal of the Peripheral Nervous System, vol. 4, no. 1, pp. 43-52, 1999.

[15] J. E. Olerud, D. S. Chiu, M. L. Usui, N. S. Gibran, and J. C. Ansel, "Protein gene product 9.5 is Expressed by fibroblasts in human cutaneous wounds," Journal of Investigative Dermatology, vol. 111, no. 4, pp. 565-572, 1998.

[16] E. L. Sidebotham, M. N. Woodward, S. E. Kenny, D. A. Lloyd, C. R. Vaillant, and D. H. Edgar, "Assessment of protein gene product 9.5 as a marker of neural crest-derived precursor cells in the developing enteric nervous system," Pediatric Surgery International, vol. 17, no. 4, pp. 304-307, 2001.

[17] B. Genç, A. K. B. Lagrimas, P. Kuru et al., "Visualization of sensory neurons and their projections in an upper motor neuron reporter line," PLoS ONE, vol. 10, no. 7, Article ID e0132815, 2015.

[18] M. Talagas, N. Lebonvallet, R. Leschiera, P. Marcorelles, and L. Misery, "What about physical contacts between epidermal keratinocytes and sensory neurons?" Experimental Dermatology, vol. 27, no. 1, pp. 9-13, 2018.

[19] E. L. Foster, E. L. Simpson, L. J. Fredrikson et al., "Eosinophils increase neuron branching in human and murine skin and in vitro," PLoS ONE, vol. 6, no. 7, Article ID e22029, 2011.

[20] L.-J. Zhang, G. L. Sen, N. L. Ward et al., "Antimicrobial Peptide LL37 and MAVS Signaling Drive Interferon- $\beta$ Production by Epidermal Keratinocytes during Skin Injury," Immunity, vol. 45, no. 1, pp. 119-130, 2016.

[21] R. Martini and H. Willison, "Neuroinflammation in the peripheral nerve: Cause, modulator, or bystander in peripheral neuropathies?" Glia, vol. 64, no. 4, pp. 475-486, 2016.

[22] M. Ritter-Jones, S. Najjar, and K. M. Albers, "Keratinocytes as modulators of sensory afferent firing," PAIN, vol. 157, no. 4, pp. 786-787, 2016.

[23] Y. Umehara, Y. Kamata, M. Tominaga, F. Niyonsaba, H. Ogawa, and K. Takamori, "Antimicrobial peptides human LL-37 and $\beta$ defensin-3 modulate the expression of nerve elongation factors in human epidermal keratinocytes," Journal of Dermatological Science, vol. 88, no. 3, pp. 365-367, 2017.

[24] K. K. Rau, C. E. Hill, B. J. Harrison et al., "Cutaneous tissue damage induces long-lasting nociceptive sensitization and regulation of cellular stress- and nerve injury-associated genes in sensory neurons," Experimental Neurology, vol. 283, pp. 413427, 2016.

[25] S.-T. Hsieh and W.-M. Lin, "Modulation of keratinocyte proliferation by skin innervation," Journal of Investigative Dermatology, vol. 113, no. 4, pp. 579-586, 1999.
[26] D. Roggenkamp, S. Köpnick, F. Stäb, H. Wenck, M. Schmelz, and G. Neufang, "Epidermal nerve fibers modulate keratinocyte growth via neuropeptide signaling in an innervated skin model," Journal of Investigative Dermatology, vol. 133, no. 6, pp. 1620-1628, 2013.

[27] N. Stankovic, O. Johansson, and C. Hildebrand, "Increased occurrence of PGP 9.5-immunoreactive epidermal Langerhans cells in rat plantar skin after sciatic nerve injury," Cell and Tissue Research, vol. 298, no. 2, pp. 255-260, 1999.

[28] L. Wang, M. Hilliges, T. Jernberg, D. Wiegleb-Edström, and O. Johansson, "Protein gene product 9.5-immunoreactive nerve fibres and cells in human skin," Cell and Tissue Research, vol. 261, no. 1, pp. 25-33, 1990.

[29] L. K. Campbell, J. R. Thomas, L. W. Lamps, B. R. Smoller, and A. L. Folpe, "Protein Gene Product 9.5 (PGP 9.5) Is Not a Specific Marker of Neural and Nerve Sheath Tumors: An Immunohistochemical Study of 95 Mesenchymal Neoplasms," Modern Pathology, vol. 16, no. 10, pp. 963-969, 2003.

[30] M. Tsutsumi, M. Nakatani, J. Kumamoto, S. Denda, and M. Denda, "In vitro formation of organized structure between keratinocytes and dorsal-root-ganglion cells," Experimental Dermatology, vol. 21, no. 11, pp. 886-888, 2012.

[31] S. M. Ostrowski, A. Belkadi, C. M. Loyd, D. Diaconu, and N. L. Ward, "Cutaneous denervation of psoriasiform mouse skin improves acanthosis and inflammation in a sensory neuropeptide-dependent manner," Journal of Investigative Dermatology, vol. 131, no. 7, pp. 1530-1538, 2011.

[32] C. Bivik Eding and C. Enerbäck, "Involved and uninvolved psoriatic keratinocytes display a resistance to apoptosis that may contribute to epidermal thickness," Acta DermatoVenereologica, vol. 97, no. 7, pp. 788-796, 2017.

[33] T. H. Zhu, M. Nakamura, B. Farahnik et al., "The Role of the Nervous System in the Pathophysiology of Psoriasis: A Review of Cases of Psoriasis Remission or Improvement Following Denervation Injury," American Journal of Clinical Dermatology, vol. 17, no. 3, pp. 257-263, 2016.

[34] M. Komine, M. Karakawa, T. Takekoshi et al., "Early inflammatory changes in the "perilesional skin" of psoriatic plaques: Is there interaction between dendritic cells and keratinocytes?" Journal of Investigative Dermatology, vol. 127, no. 8, pp. 19151922, 2007.

[35] W. Ding, L. L. Stohl, L. Xu et al., "Calcitonin gene-related peptide-exposed endothelial cells bias antigen presentation to CD4+ T Cells toward a Th17 response," The Journal of Immunology, vol. 196, no. 5, pp. 2181-2194, 2016.

[36] A. Sebastian, S. W. Volk, P. Halai, J. Colthurst, R. Paus, and A. Bayat, "Enhanced Neurogenic Biomarker Expression and Reinnervation in Human Acute Skin Wounds Treated by Electrical Stimulation," Journal of Investigative Dermatology, vol. 137, no. 3, pp. 737-747, 2017.

[37] S. H. Graham, "Modification of ubiquitin C-terminal hydrolase L1 by reactive lipid species: Role in neural regeneration and diseases of aging," Neural Regeneration Research, vol. 11, no. 6, pp. 908-909, 2016.

[38] W. R. Swindell, M. K. Sarkar, Y. Liang et al., "RNA-seq identifies a diminished differentiation gene signature in primary monolayer keratinocytes grown from lesional and uninvolved psoriatic skin," Scientific Reports, vol. 7, no. 1, 2017.

[39] G. Melino, E. Gallagher, R. I. Aqeilan et al., "Itch: A HECT-type E3 ligase regulating immunity, skin and cancer," Cell Death \& Differentiation, vol. 15, no. 7, pp. 1103-1112, 2008. 
[40] W. L. Perry, C. M. Hustad, D. A. Swing, T. Norene O’Sullivan, N. A. Jenkins, and N. G. Copeland, "The itchy locus encodes a novel ubiquitin protein ligase that is disrupted in a $(18 \mathrm{H})$ mice," Nature Genetics, vol. 18, no. 2, pp. 143-146, 1998.

[41] E. Le Pape, T. Passeron, A. Giubellino, J. C. Valencia, R. Wolber, and V. J. Hearing, "Microarray analysis sheds light on the dedifferentiating role of agouti signal protein in murine melanocytes via the Mclr," Proceedings of the National Acadamy of Sciences of the United States of America, vol. 106, no. 6, pp. 1802-1807, 2009.

[42] E. Y. Seo, S.-P. Jin, K.-C. Sohn, C.-H. Park, D. H. Lee, and J. H. Chung, "UCHL1 Regulates Melanogenesis through Controlling MITF Stability in Human Melanocytes," Journal of Investigative Dermatology, vol. 137, no. 8, pp. 1757-1765, 2017.

[43] A. L. Cicero, C. Delevoye, F. Gilles-Marsens et al., "Exosomes released by keratinocytes modulate melanocyte pigmentation," Nature Communications, vol. 6, article no. 7506, 2015.

[44] M. Cichorek, M. Wachulska, A. Stasiewicz, and A. Tymińska, "Skin melanocytes: biology and development," Postepy Dermatologii i Alergologii, vol. 30, no. 1, pp. 30-41, 2013.

[45] M. B. Abdel-Naser, A. I. Liakou, R. Elewa, S. Hippe, J. Knolle, and C. C. Zouboulis, "Increased Activity and Number of Epidermal Melanocytes in Lesional Psoriatic Skin," Dermatology, vol. 232, no. 4, pp. 425-430, 2016.

[46] C. Cheng, G. F. Guo, J. A. Martinez, V. Singh, and D. W. Zochodne, "Dynamic plasticity of axons within a cutaneous milieu," The Journal of Neuroscience, vol. 30, no. 44, pp. 1473514744, 2010.

[47] J. Wulfänger, K. Biehl, A. Tetzner et al., "Heterogeneous expression and functional relevance of the ubiquitin carboxylterminal hydrolase L1 in melanoma," International Journal of Cancer, vol. 133, no. 11, pp. 2522-2532, 2013.

[48] H. Shen, M. Sikorska, J. Leblanc, P. R. Walker, and Q. Y. Liu, "Oxidative stress regulated expression of ubiquitin Carboxylterminal Hydrolase-L1: role in cell survival," Apoptosis, vol. 11, no. 6, pp. 1049-1059, 2006.

[49] R. Karim, B. Tummers, C. Meyers et al., "Human Papillomavirus (HPV) Upregulates the Cellular Deubiquitinase UCHL1 to Suppress the Keratinocyte's Innate Immune Response," PLoS Pathogens, vol. 9, no. 5, Article ID e1003384, 2013.

[50] A. Bheda, J. Shackelford, and J. S. Pagano, "Expression and functional studies of ubiquitin C-terminal hydrolase L1 regulated genes," PLoS ONE, vol. 4, no. 8, Article ID e6764, 2009.

[51] L. Yang, W. Guo, S. Zhang, and G. Wang, "Ubiquitinationproteasome system: A new player in the pathogenesis of psoriasis and clinical implications," Journal of Dermatological Science, vol. 89, no. 3, pp. 219-225, 2018.

[52] M. Nakamura, M. Toyoda, and M. Morohashi, "Pruritogenic mediators in psoriasis vulgaris: comparative evaluation of itchassociated cutaneous factors," British Journal of Dermatology, vol. 149, no. 4, pp. 718-730, 2003.

[53] K. Taneda, M. Tominaga, O. Negi et al., "Evaluation of epidermal nerve density and opioid receptor levels in psoriatic itch," British Journal of Dermatology, vol. 165, no. 2, pp. 277-284, 2011.

[54] O. Johansson, S.-W. Han, and A. Enhamre, "Altered cutaneous innervation in psoriatic skin as revealed by PGP 9.5 immunohistochemistry," Archives of Dermatological Research, vol. 283, no. 8, pp. 519-523, 1991.

[55] S. Pergolizzi, M. Vaccaro, L. Magaudda et al., "Immunohistochemical study of epidermal nerve fibres in involved and uninvolved psoriatic skin using confocal laser scanning microscopy,"
Archives of Dermatological Research, vol. 290, no. 9, pp. 483-489, 1998.

[56] H. El-Nour, A. Santos, M. Nordin et al., "Neuronal changes in psoriasis exacerbation," Journal of the European Academy of Dermatology and Venereology, vol. 23, no. 11, pp. 1240-1245, 2009.

[57] T.-W. Kim, W.-H. Shim, J.-M. Kim et al., "Clinical characteristics of pruritus in patients with scalp psoriasis and their relation with intraepidermal nerve fiber density," Annals of Dermatology, vol. 26, no. 6, pp. 727-732, 2014.

[58] M. Krzanowska, K. Muszer, K. Chabowski, and A. Reich, "Assessment of the sensory threshold in patients with atopic dermatitis and psoriasis," Postepy Dermatologii i Alergologii, vol. 32, no. 2, pp. 94-100, 2015.

[59] E. M. J. Peters, A. Michenko, J. Kupfer et al., "Mental stress in atopic dermatitis - Neuronal plasticity and the cholinergic system are affected in atopic dermatitis and in response to acute experimental mental stress in a randomized controlled pilot study," PLoS ONE, vol. 9, no. 12, Article ID el13552, 2014.

[60] M. S. Al'Abadie, G. G. Kent, and D. J. Gawkrodger, "The relationship between stress and the onset and exacerbation of psoriasis and other skin conditions," British Journal of Dermatology, vol. 130, no. 2, pp. 199-203, 1994. 


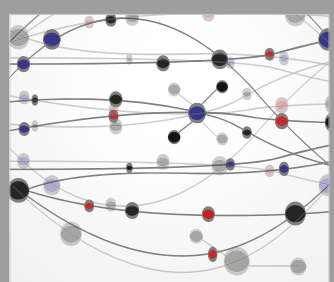

The Scientific World Journal
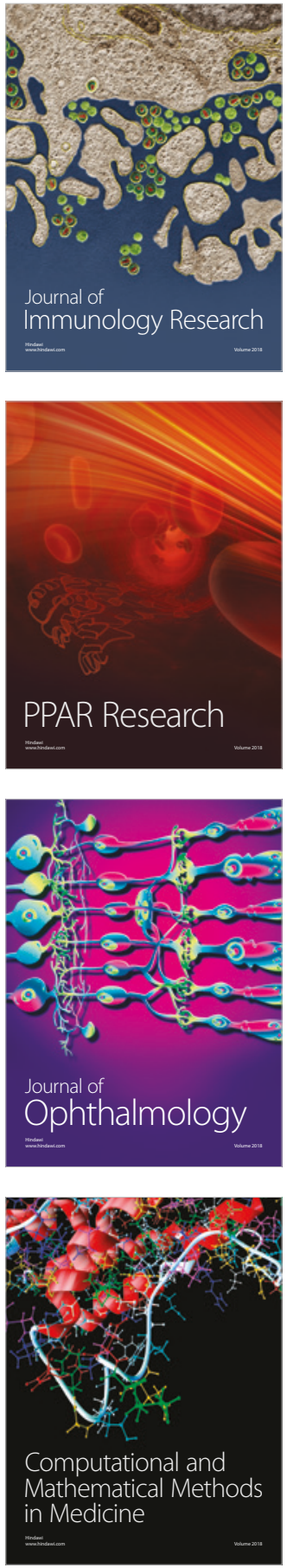

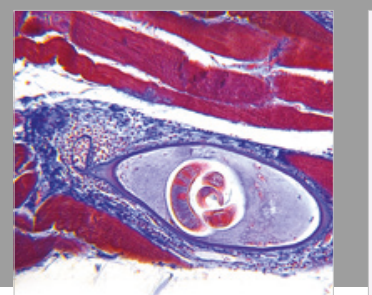

Gastroenterology Research and Practice

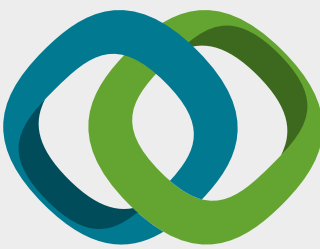

\section{Hindawi}

Submit your manuscripts at

www.hindawi.com
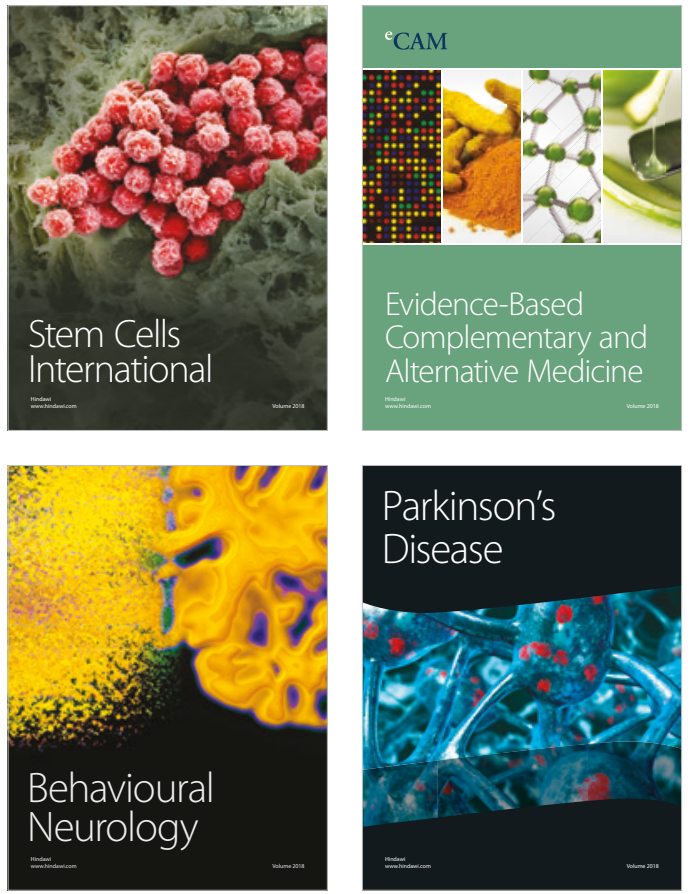

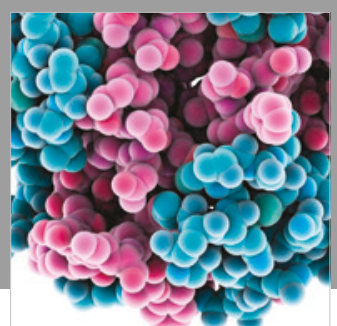

ournal of

Diabetes Research

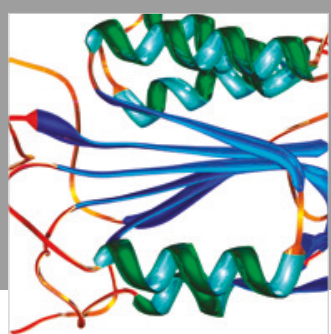

Disease Markers
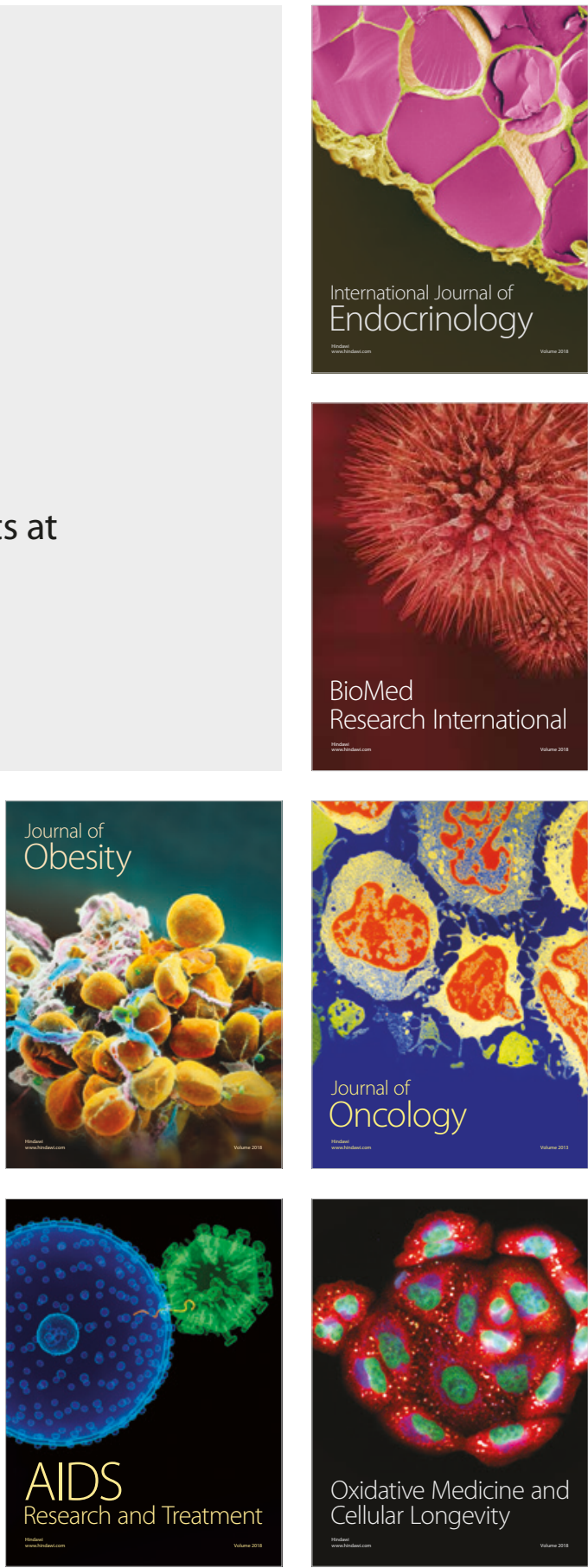